\title{
Características de la canal y de la carne de corderos de un sistema intensivo
}

\section{Carcass and meat characteristics of lamb under an intensive feeding system}

\section{Características da carcaça e da carne de cordeiros criados em um sistema intensivo}

\section{Esmeralda Desdémona Martínez ${ }^{1}$}

1 Programa de Posgrado en Producción Animal, Facultad de Zootecnia, Universidad Autónoma de Chihuahua, México. desdemona_esme@yahoo.com.mx

Recibido: 3 de octubre de 2019 Corregido: 17 de diciembre de 2019 Aceptado: 7 de enero de 2020

\begin{abstract}
Resumen
El objetivo de este trabajo es determinar las características de la canal y de la carne de corderos de diferentes fenotipos. Blackbelly (BB; $n=22$ ), Pelibuey Blanco (PB; $n=16)$, Pelibuey Canelo (PC; $n=22)$, Dorper (DO; n=37), Katahdin (KA; n=29), Pelibuey x Katahdin (PK; n=8) y Media Lana (ML; n=11), con un rango de edad entre 6 a 8 meses y criados en un sistema de finalización intensivo por $90 \mathrm{~d}$. Las variables evaluadas en la canal son: rendimiento (REND), conformación (CONF), cobertura de grasa en la canal (CG), medidas morfométricas, color de la grasa de cobertura (CGC) y color de la carne (CC). Los datos se analizan bajo un modelo de comparación de medias. Los DO, ML y KA tuvieron mejor relación en la estructura esquelética y crecimiento de los músculos y CG $(\mathrm{P}<0,05)$, que los $\mathrm{PB}, \mathrm{PC}$ y $\mathrm{BB}$, con menor peso al sacrificio y $\mathrm{CG}$. Las medidas morfométricas de la canal muestran que, los DO y ML son compactas y gruesas, resultado de la madurez temprana en la estructura esquelética, en comparación a los $\mathrm{BB}, \mathrm{PB}$ y $\mathrm{PK}$, que son más largas y tuvieron menor compacidad de la canal. Los DO presentaron un color de grasa blanca a cremosa y los BB y ML de blanca a amarilla. Los PB y DO tuvieron un color de la carne rojizo rosado y los PK rojo intenso. En conclusión, la madurez fisiológica de los diferentes fenotipos de corderos se reflejó en el REND, CONF, medidas morfométricas y CG. Por otra parte, las características propias del sistema de alimentación reflejaron variación en el CGC.
\end{abstract}

Palabras clave: fenotipo, estructura, grasa, madurez, color.

\begin{abstract}
The objective of this paper was to determinate carcass and meat characteristics of different lamb phenotypes, including: Blackbelly (BB; $n=22$ ), Pelibuey Blanco (PB; $n=16$ ), Pelibuey Canelo (PC; $n=22)$, Dorper (DO; $n=37)$, Katahdin (KA; $n=29)$, Pelibuey $x$ Katahdin $(P K ; n=8)$ and Hair $x$ Wool $(H W ; n=11)$, aged 6 to 8 months old and raised under a $90 \mathrm{~d}$ intensive finishing system. Variables assessed in the lamb carcass were yield $(\mathrm{Y})$, conformation (CONF), fat cover (FC), morphometric measurements, fat cover color (FCC) and meat color (MC). Data was analyzed using a mean comparison model. DO, HW, and KA showed better relation between skeletal structure, muscle growth, and FC $(\mathrm{P}<0.05)$ than $\mathrm{PB}, \mathrm{PC}$, and $\mathrm{BB}$, which had lower slaughter weight and FC. Carcass morphometric measurements showed that DO and HW were compact and thick, as a result of early maturity of the skeletal structure, compared to $\mathrm{BB}, \mathrm{PB}$, and $\mathrm{PK}$, which were longer and had less carcass compactness. DO's fat color was between white and beige, while BB and HW's was between white and yellow. PB and DO's meat color was reddish pink, while PK's was bright red. In conclusion, the physiological
\end{abstract}


maturity of the different lamb phenotypes is reflected in Y, CONF, morphometric measurements, and FC. On the other hand, the feeding system characteristics showed variation in FCC.

Keywords: phenotype, structure, fat, maturity, color.

\begin{abstract}
Resumo
O objetivo deste trabalho foi determinar as características da carcaça e da carne de cordeiros de diferentes fenótipos. Blackbelly (BB; $n=22$ ), Pelibuey branco (PB; $n=16$ ), Pelibuey Canelo (PC; $n=22)$, Dorper (DO; $\mathrm{n}=37$ ), Katahdin (KA; $\mathrm{n}=29$ ), Pelibuey $\mathrm{x}$ Katahdin $(\mathrm{PK} ; \mathrm{n}=8$ ) e Media Lana (ML; $\mathrm{n}=11$ ), com uma faixa etária entre 6 a 8 meses e criados em sistema de terminação intensivo por 90 dias. As variáveis avaliadas na carcaça foram: rendimento (REND), conformação (CONF), cobertura de gordura na carcaça (CG), medidas morfométricas, cor da cobertura de gordura (CGC) e cor da carne (CC). Os dados foram analisados sob um modelo de comparação de médias. O DO, ML e KA tiveram uma melhor relação na estrutura esquelética e crescimento dos músculos e $\mathrm{CG}(\mathrm{P}<0,05)$, do que $\mathrm{PB}, \mathrm{PC}$ e $\mathrm{BB}$, com menor peso no abate e CG. As medidas morfométricas da carcaça mostram que o OD e o ML são compactas e espessas, resultado da maturidade precoce na estrutura esquelética, comparados aos $\mathrm{BB}, \mathrm{PB}$ e $\mathrm{PK}$, que são mais longas e com menor compactação da carcaça. O DO mostrou uma cor de gordura que variou de branca a bege e os BB e ML de branca a amarela. Os PB e DO mostraram uma cor de carne rosa avermelhada e PK vermelho intenso. Em conclusão, a maturidade fisiológica dos diferentes fenótipos de cordeiros foi refletida nas medidas de REND, CONF, morfométricas e CG. Por outro lado, as características próprias do sistema de alimentação refletiram variação no CGC.
\end{abstract}

Palavras-chave: fenótipo, estrutura, gordura, maturidade, cor.

\title{
Introducción
}

En México, existe 8.9 millones de cabezas de ovinos (SIAP 2017). Anualmente se sacrifica 3,026,005 borregos y se obtiene 61,606 toneladas de carne en canal. De los ovinos sacrificados, $82 \%$ corresponde a machos y $18 \%$ a hembras (Partida de la Peña et al. 2017; Martínez 2019). De los primeros, el 92\% concierne a corderos no mayores de 11 meses, principalmente de los fenotipos de Dorper, Pelibuey y Katahdin (Martínez 2018). Estos son procesados para la obtención de productos tradicionales como: barbacoa, mixiote y birria. También, se ha establecido un nuevo mercado con base en cortes con hueso, como: chuleta doble o sencilla, rack francés, shoulder y medallón de cordero, empacados al alto vacío, así como productos procesados, rápidos de preparar, tales como: hamburguesa empacada en atmósfera modificada $\left(\mathrm{CO}_{2}\right.$ y Oxígeno), salchicha, chistorra, chorizo y marinados.

Las preferencias de los compradores apuntan hacia canales con poca grasa, riñonada amplia, piernas gruesas y cortas, cuello corto y ancho, frente a las canales estrechas y alargadas. Con estas bases, el trabajo se enfocó en la evaluación de corderos de más de $38 \mathrm{~kg}$ de peso vivo (PV), por la mayor incidencia de sacrificio en las plantas procesadoras en el país. Se apoya en las variables, peso de la canal caliente (PCC), rendimiento verdadero (REND) resultado del $\mathrm{PCC} / \mathrm{PV}^{\star} 100$, conformación (CONF), cobertura de grasa (CG) y medidas morfométricas; tomando como base los criterios establecidos por diferentes autores (Hunsley et al. 1978; Berg \& Butterfield 1979; Kempster et al. 1982; Swatland 1991; Delfa 1996; Boggs et al. 1998; Lawrence \& Fowler 2002; de Azeredo et al. 2006). El color de la carne (CC) y de la grasa de cobertura (CGC); fueron considerados porque influirán sobre la calidad de los cortes al detalle. Por tanto, en la aceptación por el consumidor en el centro comercial (Albertí et al. 
1993; Albertí et al.1999; da Silveira \& Moreira 2006). Además, si la planta procesadora, en un futuro pretende la exportación de carne, estos serán un punto crítico considerable. Por tanto, el objetivo del trabajo es determinar las características de la canal y de la carne de corderos, de diferente fenotipo, de un sistema intensivo.

\section{Materiales y métodos}

145 corderos, con edades entre 6 a 8 meses, criados en un sistema de finalización intensiva por 90 d, con base en concentrados (maíz y/o sorgo, pasta de soya), forraje (heno de alfalfa y/o silo de maíz) y sales minerales, fueron sacrificados en una planta procesadora de ovinos Tipo Inspección Federal, ubicada en la región centro de México, de acuerdo con las Normas Oficiales en México (NOM-009-Z00 1994; NOM-033-SAG/200 2014). Después de 18 h de refrigeración, post-sacrificio, la calidad de las canales fueron evaluadas y caracterizadas por cuatro factores: CONF, CG, medidas morfométricas, CC y CGC.

Evaluación subjetiva de la CONF. Se clasifica en: suprema (6), excelente (5), muy buena (4), buena (3), menos buena (2) e inferior (1) (SEUMIX). La valoración se sustentada de acuerdo con los criterios establecidos por Kempster et al. (1982) y Martínez et al. (2007).

Evaluación subjetiva de la CG. Se clasifica en: muy importante (5), importante (4), media (3), escasa (2) y nula (1) (Martínez 2007).

Evaluación subjetiva del CGC. Se clasifica en: blanca (6), blanca cremosa (5), blanca amarilla (4), cremosa (3), cremosa amarilla (2) y amarilla (1) de acuerdo con el patrón fotográfico que se muestra en la Figura 1 (Martínez 2014).

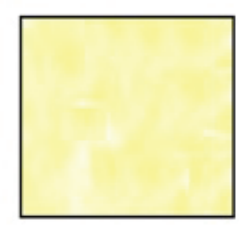

(1) AMARILLA

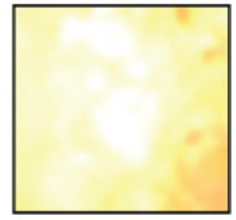

(4) BLANCA A AMARILLA



(2) CREMOSA A AMARILLA

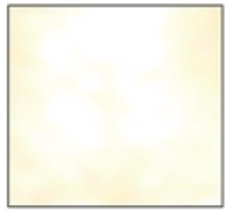

(5) BLANCA A CREMOSA

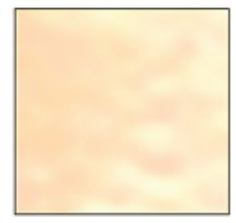

(3) CREMOSA

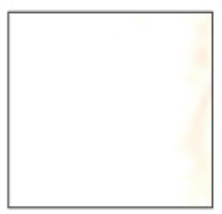

(6)BLANCA

Figura 1. Patrón fotográfico del color subjetivo de la grasa de cobertura en canales de cordero. 
Medidas morfométricas (cm). Se midió el perímetro de la grupa (PG), ancho de la grupa (AG), ancho del tórax (AT), perímetro del tórax (PT), largo de la canal (LC) y largo de la pierna (LP) y compacidad de la canal (PCC kg /LC cm) (Delfa 1996).

Evaluación objetiva del CC y CGC. El color de la carne fue medido en el músculo Obliquus internus abdominis de acuerdo con lo descrito por Boggs et al. (1998) y Martínez (2007), con un espectrofotómetro Minolta (CM-2002, Minolta Camera, Japonesa) y se determinan las coordenadas del espacio Cielab mediante sus coordenadas $\mathrm{L}^{*}, \mathrm{a}^{*} \mathrm{y} \mathrm{b}^{*}$. El color de la grasa fue medido sobre la porción posterior del lomo a nivel de la sexta vértebra lumbar.

Análisis estadístico. El análisis de los datos se realizó por el procedimiento GLM, con el paquete estadístico (SAS system ${ }^{\circledast}$ version 9 Institute, Inc., Cary, NC 2002); se estimaron las medias corregidas por mínimos cuadrados (LSMEANS), con los cálculos correspondientes, errores estándares (STERR) y comparación de medias con la prueba de Tukey.

\section{Resultados y discusión}

Los resultados indicaron (Cuadro 1), que los DO, ML y KA tuvieron mejor relación en la estructura esquelética y el desarrollo de los músculos y cobertura de grasa y se reflejó en el PV, REND y CONF, en comparación a los fenotipos $\mathrm{PB}, \mathrm{PC}$ y $\mathrm{BB}(\mathrm{P}<0,05)$, que fueron más tardíos en alcanzar el $\mathrm{PV}$ al sacrificio. Sin embargo, los DO y KA obtuvieron mayor grado CG, característica, que probablemente pueda influir en el valor comercial de los cortes primarios, pues los consumidores, en el centro del país, prefieren carnes con menos cantidad de grasa.

Cuadro 1. Medias de mínimos cuadrados de las características de las canales de corderos de diferente fenótipo.

\begin{tabular}{|c|c|c|c|c|c|c|c|}
\hline & $\begin{array}{c}B B \\
n=22\end{array}$ & $\begin{array}{c}P B \\
n=16\end{array}$ & $\begin{array}{c}\mathrm{PC} \\
\mathrm{n}=22\end{array}$ & $\begin{array}{c}\text { DO } \\
n=37\end{array}$ & $\begin{array}{c}\mathrm{KA} \\
\mathrm{n}=29\end{array}$ & $\begin{array}{c}\mathrm{PK} \\
\mathrm{n}=8\end{array}$ & $\begin{array}{c}\mathrm{ML} \\
\mathrm{n}=11\end{array}$ \\
\hline PESO VIVO (kg) & $44,27^{\mathrm{b}} \pm 0,8$ & $38,96^{c} \pm 0,9$ & $44,40^{\mathrm{b}} \pm 0,8$ & $47,97^{\mathrm{a}} \pm 0,6$ & $47,13^{\mathrm{a}} \pm 0,7$ & $42,43^{b} \pm 1,3$ & $47,36^{\mathrm{a}} \pm 1,1$ \\
\hline PESO EN CANAL $(\mathrm{kg})$ & $22,34^{\mathrm{b}} \pm 0,5$ & $19,81^{\mathrm{c}} \pm 0,6$ & $23,19^{\mathrm{b}} \pm 0,5$ & $25,45^{\mathrm{a}} \pm 0,4$ & $25,03^{\mathrm{a}} \pm 0,4$ & $21,18^{\mathrm{b}} \pm 0,9$ & $25,29^{\mathrm{a}} \pm 0,7$ \\
\hline RENDIMIENTO (\%) & $50,32^{\mathrm{b}} \pm 0,59$ & $50.86^{\mathrm{b}} \pm 0,6$ & $52,11^{\mathrm{a}} \pm 0,5$ & $53,02^{\mathrm{a}} \pm 0,46$ & $53,08^{a} \pm 0,5$ & $49,85^{b c} \pm 0,9$ & $53,38^{\mathrm{a}} \pm 0,8$ \\
\hline CONFORMACIÓN* & $3,22^{\mathrm{b}} \pm 0,17$ & $3,25^{b} \pm 0,19$ & $3,59^{\mathrm{ab}} \pm 0,17$ & $4,05^{\mathrm{bc}} \pm 0,13$ & $4,10^{\mathrm{bc}} \pm 0,1$ & $3,75^{\mathrm{ab}} \pm 0,2$ & $3,90^{c} \pm 0,2$ \\
\hline $\begin{array}{l}\text { GRASA DE } \\
\text { COBERTURA** }^{*}\end{array}$ & $3,50^{\mathrm{ab}} \pm 0,18$ & $3,12^{\mathrm{b}} \pm 0,2$ & $3,68^{\mathrm{a}} \pm 0,18$ & $4,11^{\mathrm{c}} \pm 0,14$ & $4,10^{c} \pm 0,1$ & $4,00^{c} \pm 0,2$ & $3,72^{\mathrm{a}} \pm 0,2$ \\
\hline
\end{tabular}

Media \pm Error estándar

a, b, c Medias en el mismo renglón con diferente literal son diferentes $(\mathrm{P}<0,05)$

${ }^{*}$ CONF. Conformación subjetiva evaluada en 6 clases (SEUMIX); Suprema (6), excelente (5), muy buena (4), buena (3), menos buena (2) e inferior (1).

${ }^{*}$ CG. Cobertura de grasa subjetiva evaluada en 5 clases; muy importante (5), importante (4), media (3), escasa (2) y nula (1).

El rendimiento obtenido de los DO $(53,02 \%)$ y KA $(53,08 \%)$ fue superior a lo reportado en corderos de 7 meses (Burke \& Apple 2007), con un menor PV al sacrificio de 38,4 kg, DO (51\%) y KA (47,1\%). Estos 
autores concluyen que los DO tuvieron mayor desarrollo muscular con poca grasa a temprana edad, en comparación con los KA. Burke et al. (2003). Reportaron que, en corderos cruzados de DO x Saint Croix, las canales fueron más pesadas; tuvieron mayor grado de CONF, CG, REND, área del Longissimus dorsi thoracis, al compararlos con corderos puros KA, Saint Croix y 3/4 Saint Croix x 1/4 Romanov.

También, se reporta que los corderos DO tienen altas velocidades de crecimiento; por tanto, obtienen un peso de sacrificio más temprano que otras razas de pelo (Snowder et al. 1994; Schackelford et al. 2005). En los sistemas productivos es importante tomar en cuenta la habilidad propia de un cordero en la eficiencia alimenticia y ganancia de peso sobre la madurez estructural esquelética y la composición de tejidos (músculo y grasa) sobre ésta. Posiblemente, si la planta procesadora sacrifica corderos DO y $\mathrm{KA}$, en un rango de PV entre 40 y $43 \mathrm{~kg}$, se obtenga canales con menos grados de CG, sin detrimento en los grados de CONF y REND de los cortes primarios.

Sin embargo, Fahmy (1996) y Greiner \& Ducket (2006) reportaron que los corderos KA producen canales más pesadas $(20,13 \mathrm{~kg})$ con mayor CG que los BB y Saint Croix.

Respecto a los corderos PC, se reportan valores similares (Gutiérrez \& Méndez 2005) a los de este trabajo, en cuanto a rendimiento (44,49 vs 44,40\%). También, mencionan que tuvieron menor CG que los PC cruzados con Suffolk y Romanov y no difirieron en el REND y área del ojo de la costilla. Estos autores concluyen que, la inclusión de Romanov, en el componente genotipo de los PC, no es recomendable, de acuerdo con las preferencias del mercado mexicano, por menos cantidad de grasa intramuscular.

Por consiguiente, de acuerdo con los resultados obtenidos en REND, CONF y CG, los PC, se están sacrificando en un PV aceptable, de acuerdo con las preferencias del mercado en el centro del país. En los $\mathrm{BB}$ y $\mathrm{PB}$, el peso ideal sería en un rango de 45-48 kg. Sin embargo, en los corderos ML es necesario realizar una evaluación de las canales según los programas de cruzamiento (lana y pelo) que están empleando en los sistemas productivos dirigidos a la producción de carne, pues los resultados mostrados en este trabajo presentan características semejantes a los KA y DO.

Referente a las medidas morfométricas de la canal, el LC fue mayor en los PK $(52,00 \mathrm{~cm})(\mathrm{P}<0,05)$ y menor en los PC (47,27 cm), estos últimos fueron iguales $(\mathrm{P}>0,05)$ a los demás fenotipos (Cuadro 2). Los $\mathrm{ML}$, tuvieron mayor LP $(36,37 \mathrm{~cm})(\mathrm{P}<0,05)$ que los $\mathrm{PK}(30,20 \mathrm{~cm})$ y estos últimos iguales $(\mathrm{P}>0,05)$ a los demás fenotipos. Cloete et al. (2000) reportaron $0,42 \mathrm{~cm}$ y 0,62 $\mathrm{cm}$ menos en la LC y LP, en corderos PC que en aquellos cruzados con animales de lana.

En cuanto al PT, los PB tuvieron menor medida $(69,00 \mathrm{~cm}) \mathrm{P}<0,05)$ que los $\mathrm{ML}(77,40 \mathrm{~cm}),. \mathrm{DO}(77,58 \mathrm{~cm})$ y KA $(75,77)$. Además, el AT fue menor en los $\mathrm{BB}$ y $\mathrm{PB}(\mathrm{P}<0,05)$ que los otros fenotipos, características que probablemente tuvieron influencia en el PCC, REND y CONF (Cuadro 1), pues diversos autores han reportado que el PV tiene gran influencia sobre las medidas de la canal (Sañudo et al. 1993; Rojas 2002; da Silveira \& Moreira 2006). 
Cuadro 2. Medias de mínimos cuadrados de las medidas morfométricas $(\mathrm{cm})$ en canales de corderos de diferente fenotipo.

\begin{tabular}{lccccccc}
\hline & $\mathrm{BB}$ & $\mathrm{PB}$ & $\mathrm{PC}$ & $\mathrm{DO}$ & $\mathrm{KA}$ & $\mathrm{PK}$ & $\mathrm{ML}$ \\
& $\mathrm{n}=22$ & $\mathrm{n}=16$ & $\mathrm{~N}=22$ & $\mathrm{n}=37$ & $\mathrm{n}=29$ & $\mathrm{n}=8$ & $\mathrm{n}=11$ \\
& & & & & & \\
\hline $\begin{array}{l}\text { Largo de la } \\
\text { Canal }(\mathrm{cm})\end{array}$ & $49,04^{\mathrm{a}} \pm 0,83$ & $49,03^{\mathrm{a}} \pm 0,74$ & $47,27^{\mathrm{ab}} \pm 0,92$ & $48,45^{\mathrm{a}} \pm 0,80$ & $48,95^{\mathrm{a}} \pm 0,83$ & $52,00^{\mathrm{c}} \pm 0,97$ & $47,40^{\mathrm{a}} \pm 1,23$ \\
$\begin{array}{l}\text { Longitud de la } \\
\text { Pierna }(\mathrm{cm})\end{array}$ & $32,54^{\mathrm{a}} \pm 0,68$ & $32,35^{\mathrm{a}} \pm 0,60$ & $31,38^{\mathrm{a}} \pm 0,75$ & $32,04^{\mathrm{a}} \pm 0,65$ & $31,95^{\mathrm{a}} \pm 0,68$ & $30,20^{\mathrm{a}} \pm 1,01$ & $36,37^{\mathrm{b}} \pm 0,80$ \\
$\begin{array}{l}\text { Perímetro del } \\
\text { Tórax }(\mathrm{cm})\end{array}$ & $74,09^{\mathrm{c}} \pm 1,03$ & $69,00^{\mathrm{b}} \pm 0,91$ & $74,77^{\mathrm{c}} \pm 1,14$ & $77,58^{\mathrm{a}} \pm 0,99$ & $75,77^{\mathrm{c}} \pm 1,03$ & $73,50^{\mathrm{c}} \pm 1,21$ & $77,40^{\mathrm{a}} \pm 1,53$ \\
$\begin{array}{l}\text { Anchura del } \\
\text { Tórax }(\mathrm{cm})\end{array}$ & $21,77^{\mathrm{b}} \pm 0,74$ & $21,32^{\mathrm{b}} \pm 0,66$ & $23,38^{\mathrm{a}} \pm 0,82$ & $23,79^{\mathrm{a}} \pm 0,71$ & $23,50^{\mathrm{a}} \pm 0,74$ & $23,75^{\mathrm{a}} \pm 0,87$ & $24,60^{\mathrm{a}} \pm 1,11$ \\
$\begin{array}{l}\text { Perímetro de la } \\
\text { grupa }(\mathrm{cm})\end{array}$ & $59,72^{\mathrm{a}} \pm 0,50$ & $58,10^{\mathrm{a}} \pm 0,84$ & $62,38^{\mathrm{ab}} \pm 1,01$ & $62,29^{\mathrm{ab}} \pm 0,88$ & $62,45^{\mathrm{ab}} \pm 0,92$ & $69,93^{\mathrm{ac}} \pm 1,07$ & $63,90^{\mathrm{a}} \pm 1,36$ \\
$\begin{array}{l}\text { Anchura de la } \\
\text { grupa }(\mathrm{cm})\end{array}$ & $18,00^{\mathrm{a}} \pm 0,50$ & $16,82^{\mathrm{c}} \pm 0,44$ & $18,44^{\mathrm{a}} \pm 0,56$ & $20,41^{\mathrm{b}} \pm 0,48$ & $17,80^{\mathrm{c}} \pm 0,50$ & $18,00^{\mathrm{a}} \pm 0,59$ & $20,20^{\mathrm{b}} \pm 0,75$ \\
$\begin{array}{l}\text { Compacidad de } \\
\text { la canal }(\mathrm{kg} / \mathrm{cm})\end{array}$ & 0,47 & 0,40 & 0,49 & 0,53 & 0,51 & 0,40 & 0,52 \\
\hline
\end{tabular}

Media \pm Error estándar

${ }^{\text {abc }}$ Medias en el mismo renglón con diferente literal son diferentes $(\mathrm{P}<0,05)$.

El PG de los PK fue mayor $(69,93 \mathrm{~cm})(\mathrm{P}<0.05)$ que los $\mathrm{BB}(59,72 \mathrm{~cm})$ y PB $(58,10 \mathrm{~cm})$. Los DO, KA, PC y ML no difirieron en esta medida $(\mathrm{P}>0.05)$. Finalmente, los $\mathrm{DO}$ y $\mathrm{ML}$ fueron mayores $(\mathrm{P}<0,05)$ en el AG $(20,41 \mathrm{~cm})$ y $(20,20 \mathrm{~cm})$ que los PB $(16,82 \mathrm{~cm})$ y KA $(17,80 \mathrm{~cm})$. En consecuencia, en la evaluación subjetiva de CONF se debe poner énfasis en la región del tórax y de la grupa, dado que, según Delfa et al. (1992), existe una clara correlación ( $\mathrm{r}=0.63)$ entre la CONF y las medidas de anchura en las regiones del tórax y de la parte posterior.

Por su parte, Sañudo et al. (1993) indican que el PG y AG contribuyen significativamente con el peso total de músculo en la canal y, probablemente, en el REND de los cortes del lomo. Estos datos se corroboran con la compacidad de la canal (PCC/LC). Los DO, ML y KA tuvieron mayor compacidad (0,53, 0,52 y $0,51 \mathrm{~kg} / \mathrm{cm})$. Los PC, BB, PB y PK tuvieron menor $(0,49,0,47,0,40$ y $0,40 \mathrm{~kg} / \mathrm{cm})$ respectivamente. Así, la diferencia en PV de los diferentes fenotipos tuvo gran influencia en las medidas morfométricas y la compacidad de la canal, confirmando lo reportado por (Sañudo et al. 1993; Santos et al. 2007).

Semejantes índices de compacidad con los DO y ML, reportaron de Azeredo et al. (2006), en corderos de lana (Corriedale), con 210 días de edad y $38 \mathrm{~kg}$ de PV (53,72 kg/cm.). Esto, tal vez, porque los últimos fueron de menor tamaño, y más largos a menor edad que los de pelo. También, Sañudo et al. (1993) reportaron que el PV tuvo más efecto que el sexo sobre los índices de compacidad y medidas morfométricas de la canal.

En la evaluación subjetiva del color de la CGC (Cuadro 3), los ML tuvieron valores de 4,54 (tendientes a blanca cremosa) y fueron diferentes $(\mathrm{P}<0,05)$ a los $\mathrm{PK} 3,0$, 
(cremosa) y PB 3,4 (cremosa-amarillo). Los PC, BB, DO y KA no mostraron diferencia (P>0.05). Así, en la evaluación objetiva del CGC, (Cuadro 3) los DO tuvieron valores más altos $\left(\mathrm{L}^{*}=65,0\right)$; los $\mathrm{PB}$ más bajos $\left(L^{*}=59.0\right)(P<0,05)$, resultado de mayor grado de engrasamiento en los primeros, que en los segundos. Los $\mathrm{BB}, \mathrm{PC}, \mathrm{KA}$ y $\mathrm{PK}$ no mostraron diferencias $(\mathrm{P}>0,05)$ atribuido a la expresión genética que dicta cada fenotipo en la deposición de grasa.

Cuadro 3. Medias de mínimos cuadrados de la evaluación subjetiva y objetiva del color de la grasa de cobertura de canales corderos de diferente fenotipo.

\begin{tabular}{|c|c|c|c|c|c|c|}
\hline $\begin{array}{c}\mathrm{BB} \\
\mathrm{n}=22\end{array}$ & $\begin{array}{c}\mathrm{PB} \\
\mathrm{n}=16\end{array}$ & $\begin{array}{c}\mathrm{PC} \\
\mathrm{n}=22\end{array}$ & $\begin{array}{c}\mathrm{DO} \\
\mathrm{n}=37\end{array}$ & $\begin{array}{c}\mathrm{KA} \\
\mathrm{n}=29\end{array}$ & $\begin{array}{c}\mathrm{PK} \\
\mathrm{n}=8\end{array}$ & $\begin{array}{c}\mathrm{ML} \\
\mathrm{n}=11\end{array}$ \\
\hline $\mathrm{CCG}^{*}=3,81^{\mathrm{a}} \pm 0,31$ & $3,43^{\mathrm{b}} \pm 0,36$ & $3,95^{a} \pm 0,31$ & $3,77^{a} \pm 0,24$ & $3,75^{a} \pm 0,27$ & $3,00^{c} \pm 0,51$ & $4,54^{\mathrm{d}} \pm 0,44$ \\
\hline$L^{*}=63,2^{a} \pm 0,85$ & $L^{*}=59,0^{b} \pm 1,0$ & $L^{\star}=61,9^{a} \pm 0,85$ & $L^{*}=65,0^{c} \pm 0,67$ & $L^{*}=62,4^{a} \pm 0,74$ & $L^{\star}=61,6^{\mathrm{a}} \pm 1,42$ & $\mathrm{~L}^{\star}=62,5^{\mathrm{a}} \pm 1,21$ \\
\hline $\mathrm{a}^{*}=5,3^{\mathrm{a}} \pm 0,75$ & $\mathrm{a}^{\star}=4,0^{\mathrm{b}} \pm 0,89$ & $\mathrm{a}^{\star}=6,3^{\mathrm{a}} \pm 0,75$ & $\mathrm{a}^{*}=5,3^{\mathrm{a}} \pm 0,59$ & $\mathrm{a}^{*}=6,8^{\mathrm{a}} \pm 0,66$ & $\mathrm{a}^{\star}=3,2^{\mathrm{b}} \pm 1,25$ & $\mathrm{a}^{\star}=8,2^{\mathrm{a}} \pm 1,07$ \\
\hline $\mathrm{b}^{*}=10,1^{\mathrm{a}} \pm 0,67$ & $b^{\star}=11,9^{a} \pm 0,79$ & $\mathrm{~b}^{\star}=8,7^{\mathrm{ab}} \pm 0,67$ & $\mathrm{~b}^{*}=10,0^{\mathrm{a}} \pm 0,53$ & $\mathrm{~b}^{*}=10,4^{\mathrm{a}} \pm 0,59$ & $b^{*}=7,9^{c} \pm 1,12$ & $\mathrm{~b}^{*}=8,3^{\mathrm{b}} \pm 0,95$ \\
\hline
\end{tabular}

Media \pm Error estándar

abc Medias en el mismo renglón con diferente literal son diferentes $(\mathrm{P}<0,05)$

CGC $^{\star}$. Color subjetivo de color de la cobertura de la grasa evaluada en 6 clases; blanca (6), blanca cremosa (5), blanca amarilla (4), cremosa (3), cremosa amarilla (2) y amarilla (1).

La determinación objetiva del color de la grasa fue medido con un espectrofotómetro Minolta. El valor L* mide la luminosidad, cuando los valores son mayores son más brillosos, tendiente a un color más blanco. El valor a* mide el matiz rojo cuando es máximo y verde cuando es mínimo. El valor b* mide el matiz amarillo cuando es máximo y azul cuando es mínimo.

Los valores de $\mathrm{a}^{*}$, fueron más altos en $\operatorname{los} \mathrm{ML}\left(\mathrm{a}^{*}=8,2\right)$; PB y PK tuvieron los valores más bajos $\left(\mathrm{a}^{*}=4,0\right.$ y 3,2$)(P<0,05)$, por mayor tiempo en alcanzar el peso de sacrificio en los segundos y más tiempo en metabolizar los betacarotenos a nivel ruminal. Sin embargo, los $\mathrm{PB}$ y KA, presentaron los valores más altos $\left(\mathrm{b}^{*}=11,9\right.$ y 10,4$)$ por menor madurez fisiológica.

Los más bajos fueron para los $\mathrm{PK}$ y ML $\left(\mathrm{b}^{*}=7,9\right.$ y 8,3) $(\mathrm{P}<0,05)$, probablemente atribuido a salpicaduras de sangre en la grasa en el área de muestreo en el momento de realizar la actividad. Al respecto Beriain et al. (2000) reportaron, en corderos de lana con $36 \mathrm{~kg}$ de PV y $131 \mathrm{~d}$ de edad, valores más altos de $\mathrm{L}^{*}$ $(68,46)$, menores de $\mathrm{a}^{*}(2,66)$ e intermedios de $\mathrm{b}^{*}(9,56)$, reflejo de mayor grado de CG en la canal y coloraciones de grasa tendientes a cremosa.

Al considerar los tres valores objetivos, en la medición del color de la grasa y la evaluación subjetiva, cuando en la práctica se observa que las canales cremosas y blancas cremosas presentaban los valores más altos de $\left(\mathrm{L}^{*}\right)$ tendientes a la claridad, valores bajos de $\left(\mathrm{a}^{*}\right)$ y valores medianamente tendientes a amarillo $\left(b^{\star}\right)$, se puede afirmar que los corderos DO presentan un color de grasa subjetivamente más blanca a cremosa y, consecuentemente, los BB y ML de blanca a amarilla. 
Cuadro 4. Medias de mínimos cuadrados de los valores del color de la carne $\left(L^{*} a^{*}\right.$ y $\left.b^{*}\right)$ de corderos de diferente fenotipo.

\begin{tabular}{ccccccc}
\hline BB & PB & PC & DO & KA & PK & ML \\
$n=22$ & $n=16$ & $n=22$ & $n=37$ & $n=29$ & $n=8$ & $n=11$ \\
\hline $\mathrm{L}^{*}=39,74^{\mathrm{a}} \pm 1,17$ & $\mathrm{~L}^{*}=36,76^{\mathrm{b}} \pm 1,37$ & $\mathrm{~L}^{*}=37,83^{\mathrm{ab}} \pm 1,17$ & $\mathrm{~L}^{*}=36,16^{\mathrm{b}} \pm 0,91$ & $\mathrm{~L}^{*}=38,58^{\mathrm{a}} \pm 1,02$ & $\mathrm{~L}^{*}=39,74^{\mathrm{a}} \pm 1,66$ & $\mathrm{~L}^{*}=45,3^{\mathrm{ac}} \pm 1,94$ \\
$\mathrm{a}=9,73^{\mathrm{b}} \pm 0,47$ & $\mathrm{a}^{*}=8,96^{\mathrm{a}} \pm 0,55$ & $\mathrm{a}^{*}=10,72^{\mathrm{c}} \pm 0,47$ & $\mathrm{a}^{*}=8,89^{\mathrm{ab}} \pm 0,37$ & $\mathrm{a}^{*}=9,97^{\mathrm{a}} \pm 0,41$ & $\mathrm{a}^{*}=7,26^{\mathrm{ab}} \pm 0,79$ & $\mathrm{a}^{*}=8,11^{\mathrm{ab}} \pm 0,67$ \\
$\mathrm{~b}=11,18^{\mathrm{a}} \pm 1,04$ & $\mathrm{~b}^{*}=9,02^{\mathrm{a}} \pm 1,22$ & $\mathrm{~b}^{*}=12,14^{\mathrm{a}} \pm 1,04$ & $\mathrm{~b}^{*}=9,99^{\mathrm{a}} \pm 0,81$ & $\mathrm{~b}^{*}=9,43^{\mathrm{a}} \pm 0,91$ & $\mathrm{~b}^{*}=3,60^{\mathrm{b}} \pm 1,73$ & $\mathrm{~b}^{*}=9,43^{\mathrm{a}} \pm 1,47$ \\
\hline
\end{tabular}

Media \pm Error estándar

abc Medias en el mismo renglón con diferente literal son diferentes $(\mathrm{P}<0,05)$

La determinación objetiva del color de la carne fue medido con un espectrofotómetro Minolta. El valor L* mide la luminosidad, cuando los valores son mayores son más brillosos, tendiente a un color más blanco. El valor $\mathrm{a}^{*}$ mide el matiz rojo cuando es máximo y verde cuando es mínimo. El valor $b^{*}$ mide el matiz amarillo cuando es máximo y azul cuando es mínimo. Valores bajos de $L^{\star}, a^{*} y b^{*}$ significa que es carne más obscura.

Los resultados reflejan que estos corderos tuvieron una alimentación con un mayor porcentaje de granos, en comparación con los otros fenotipos. Fundado en que las dietas tenían menores cantidades de b-caroteno (Swatland 1991) o combinados con la composición de los ácidos grasos; posiblemente, por una mejor eficiencia alimenticia, dado que Mora \& Shimada (2001), reportan que la tasa de desaparición de los b-caroteno, a nivel ruminal, está asociada a la tasa de desaparición de la materia seca y del contenido celular, por la actividad intestinal de la enzima 15,15' dioxigenasa.

Este factor es importante de valorar porque influirá en la calidad de los cortes al detalle y la selección, por parte de los consumidores, en el momento de la compra. También, si el objetivo de las plantas procesadoras es exportar carne, este será un punto crítico considerable.

El color de la carne, en los corderos ML (Cuadro 4), fue más luminoso $\left(\mathrm{L}^{*}=45,39\right)(\mathrm{P}<0,05)$ en comparación con los demás fenotipos. Los PB y DO tuvieron menores valores $\left(L^{*}=36,76\right.$ y 36,16$)$. Esto, tal vez, se debió a la diferencia en pigmentos hemínicos entre fenotipos, al dar como resultado diferentes espectros de absorbancia y reflactancia de luz de distintas longitudes de onda (colores). Sin embargo, Burke \& Apple (2007) reportan valores más altos de $L^{*}(33,6)$, en corderos de lana Suffolk en comparación con los de pelo, tales como; DO $\left(\mathrm{L}^{*}=32,7\right.$ y KA $\left(\mathrm{L}^{*}=31,8\right)$ pero a menor peso al sacrificio $(38,4 \mathrm{~kg})$ que, en este trabajo, esto tal vez por el efecto de la raza. Los KA y PC obtuvieron valores más rojos $\left(a^{\star}=10,72\right.$ y 9,97$)$ que los corderos $\mathrm{PK}(\mathrm{P}<0,05)\left(\mathrm{a}^{*}=7,26\right)$. Los $\mathrm{PC}$ y BB tuvieron valores más amarillos $\left(\mathrm{b}^{\star}=12,14\right.$ y 11,18$)$ en comparación con los $\mathrm{PK}(\mathrm{P}<0,05)\left(\mathrm{b}^{*}=3,60\right)$.

En general, los PB $\left(L^{*}=36,76, a^{*}=8,96, b^{*}=9,02\right), D O\left(L^{*}=36,16, a^{*}=8,89, b^{*}=9,9\right)$ y $\mathrm{PK}\left(L^{*}=39,74, a^{*}=7,26\right.$, $\mathrm{b}^{*}=3,60$ ), presentan los valores más bajos de $\mathrm{L}^{*} \mathrm{a}^{\star} \mathrm{y} \mathrm{b}^{\star}$, lo cual indica que tuvieron un color rojizo rosado para los dos primeros y rojo intenso, para los terceros, en comparación con los demás fenotipos, los cuales tuvieron coloraciones rojizo rosados y rojo claro. Probablemente, esta característica, en el color de la carne, sea una ventaja en los cortes que se expenden al detalle, pues los consumidores asocian los colores rojos intensos con carnes más frescas y saludables. En este trabajo, fue válido en los DO con mayor peso vivo, pero no concuerda con los Media lana dado que estos presentan valores de $L^{\star}$ más altos y un PV similar a los DO; quizá estos resultados se deben al efecto de la cruza con lana, Sañudo 
et al. (1993), Boggs et al. (1998), Fisher et al. (2004) y da Silveira \& Moreira (2006) han reportado que, la carne de los corderos de lana presentan valores más altos de $\mathrm{L}^{*} \mathrm{y} \mathrm{a}^{*}$.

\section{Conclusiones}

Los DO, ML y KA mostraron similar estructura esquelética y temprana madurez fisiológica; lo cual se refleja en mejores características de la canal; mientras que, los PB, BB y PK tuvieron menor peso de sacrificio, peso de la canal caliente, rendimiento y conformación. Las canales con mayor perímetro de tórax y grupa tuvieron mayor compacidad de la canal y rendimiento. El color de la grasa y carne fue muy variable entre fenotipos, atribuido, principalmente, a la alimentación, madurez fisiológica y características propias de cada fenotipo.

\section{Conflicto de interés}

Ninguno.

\section{Referencias}

Albertí, P., Sañudo, C., Santolaria, P., Negueruela, I., Consigli, R., Sierra, I., \& Alcalde, M.J. 1993. Representación en tres dimensiones en el color de la carne y grasa de bovino y su relación con otras carnes. ITEA 12 (2): 36-41.

Albertí, P., Sañudo, C., Olleta, J.L., Campo, M.M., Panea, B., Franco, J., \& Lahoz, F. 1999. Color del músculo y de la grasa subcutánea de terneros de siete encastes españolas. ITEA 20 (1): 19-25.

de Azeredo, D.M., Osório, M.T.M., Osório, J.C.S., de Mendoça, G., Esteves, R.M., Rota, E.L., Jardim, R.D. \& Pradiée, J. 2006. Morfologia in vivo e da carcaça e características produtivas e comerciais em ovinos corriedale não castrados, castrados e criptorquidas abatidos em diferentes idades. R. Bras. Agrociência 12(2): 199-204. doi: /10.18539/cast.v12i2.4525.

Berg, R.T., \& Butterfield, R.M. 1979. Nuevos conceptos sobre desarrollo de ganado vacuno: Métodos para medir y predecir la composición de la canal. Acribia, Zaragoza, España. 298p.

Beriain, M.J., Horcada, A., Purroy, A., Lizaso, G., Chasco, J. \& Mendizabal, J.A. 2000. Characteristics of Lacha and Rasa Aragonesa lambs slaughtered at three live weights. J. Anim. Sci. 78(12): 30703077. doi: $10.2527 / 2000.78123070 x$.

Boggs, D.L., Merkel, R.A., Doumit, M.E. \& Bruns, K. 1998. Livestock and carcasses. An integrated approached to evaluation, grading, and selection: Lamb carcass evaluation and grading, $5^{\text {th }}$ Edition. Kendall Hunt Publishing Company, Iowa, Estados Unidos. 258p.

Burke, J.M., Apple, J.K., Roberts, W.J., Boger, C.B. \& Kegley, E.B. 2003. Effect of breed-type on performance and carcass traits of intensively managed hair sheep. Meat Sci. 63(3):309-315. doi: 10.1016/S0309-1740(02)00087-6

Burke, J.M. \& Apple, J.K. 2007. Growth performance and carcass traits of forage-fed hair sheep wethers. 
Small Rum. Res. 67(2-3): 264-270. doi: 10.1016/j.smallrumres.2005.10.014.

Cloete, S.W.P., Snyman, M.A. \& Herselman, M.J. 2000. Productive performance of Dorper sheep. Small Ruminant Res. 36(2): 119-135. doi: 10.1016/S0921-4488(99)00156-X.

Delfa, R.A., Teixeira, A. \& González, C. 1992. Composición de la canal. Medida de composición de la canal. Fichero Ovis 23: 9-22.

Delfa, R. 1996. Predicción de la composición corporal y de la canal a partir del animal vivo y de canal. Unidad de Tecnología en Producción Animal. Fichero Ovis 23: 25-53.

Fahmy, M.H. 1996. Prolific sheep: The barbados Blackbelly. CAB International, Wallingford, UK. 560p.

Fisher, M., Duckett, S. \& Wildeus, S. 2004. Effect of breed type, finishing treatment, and dietary supplements on carcass characteristics and tenderness in hair sheep. J. Anim. Sci. 82(Suppl.1): 227.

Gutiérrez, J., Rubio, M.S. \& Méndez, R.D. 2005. Effects of crossbreeding Mexican Pelibuey sheep with Rambouillet and Suffolk on carcass traits. Meat Sci. 70(1): 1-5. doi: 10.1016/j.meatsci.2004.10.017

Hunsley, R.E., Beeson, W.M. \& Nordby, J.E. 1978. Livestock judging, selection and evaluation: Sheep carcass evaluation and judged, $2^{\text {nd }}$ Edition. The Interstate, Danville, Illinois. 527p.

Kempster, A.J., Cuthbertson, A. \& Harrington, G. 1982. Carcasses evaluation in livestock breeding, production and marketing: Carcass grading and classification. Granada Publishing, England. $306 \mathrm{p}$.

Lawrence, T.L.J. \& Fowler, V.R. 2002. Growth of farm animals: Measuring growth, $2^{\text {nd }}$ Edition. Cabiternational, Oxon, UK. 347p.

Martínez, E.D., Núñez, G.F.A. \& Rodríguez, A.F.A. 2007. Manual para la evaluación de corderos en pie y en canal. Potencial para la producción de carne. Universidad Autónoma de Chihuahua, México. 36p.

Martínez, E.D. 2014. Evaluación en pie y en canal de corderos. Trillas, México. 86p.

Martínez, E.D. 2018. Caracterización de canales de ovinos de diferente fenotipo. Entorno Ganadero 15(91):65-71. doi: 10.13140/RG.2.2.17510.47687

Martínez, E.D. 2019. Características de canales de ovinos en la región centro de México. XLVI Reunión Científica de la Asociación Mexicana Para la Producción Animal y Seguridad Alimentaria, A.C. Revista Mexicana de Agroecosistemas 6(Suppl.2): 675-682.

Mora, I.O. \& Shimada, M.A. 2001. Causas del color amarillo de la grasa de canales de bovinos finalizados en pastoreo. Vet. México 32(1): 63-71.

da Silveira, O.J.C. \& Moreira, O.M.T. 2006. Qualidade e seus determinantes na cadeia produtiva e comercial da carne ovina. R. Bras. Agrociência 12(3): 251-256. 2006. doi: 10.18539/cast.v12i3.4546.

NOM-009-200, 1994. Proceso sanitario de la carne. https:/www.gob.mx/cms/uploads/attachment/ file/203870/Mod_NOM-009-ZOO-1994_310707.pdf. 
Rev. Ciencias Veterinarias, Vol. 38, N 1, [17-27], E-ISSN: 2215-4507, enero-junio, 2020

DOI: https://doi.org/10.15359/rcv.38-1.2

Ciencias

URL: http://www.revistas.una.ac.cr/index.php/veterinaria/index

NOM-003-SAG/200, 2014. Métodos para dar muerte a los animales domésticos y silvestres. https:// www.gob.mx/profepa/documentos/norma-oficial-mexicana-nom-033-sag-zoo-2014-metodospara-dar-muerte-a-los-animales-domesticos-y-silvestres.

Partida de la Peña, J.A., Ríos, F.G., Colín, L., Domínguez, I.A. \& Buendía, G. 2017. Caracterización de las canales ovinas producidas en México. Rev. Mex. Cienc. Pec. 8(3): 269-277. doi: 10.22319/rmcp. v8i3.4203

Rojas, R.O. 2002. Reproducción y características genéticas de las principales razas de ovinos de pelo. Simposium Internacional de Ovinos. Chihuahua, México. pp 71-74.

Santos, V.A.C., Silva, S.R., Mena, E.G. \& Azevedo, J.M.T. 2007. Live weight and sex effects on carcass and meat quality of "Borrego terrincho-PDO" suckling lambs. Meat Sci. 77(4): 654-661. doi: 10.1016/j.meatsci.2007.05.019.

Sañudo, C., Sierra, I., Alcalde, M.J., Rota, A. \& Osorio, J.C.S. 1993. Calidad de la canal y de la carne en corderos semipesados de cinco encastes españolas. ITEA 3: 203-214.

SAS, 2002. User's guide statistics. Version 9 for Microsoft Windows. SAS Institute, Cary, N.C., Estados Unidos.

Greiner, S.P. \& Duckett, S.K. 2006. Fatty acid composition and palatability of lamb from hair sheep. https://www.sites.ext.vt.edu/newsletter-archive/livestock/aps-06 05/aps-328.html. 8/10/2007.

Shackelford, S.D., Leymaster, K.A., Wheeler, T.L. \& Koohmaraie, M. 2005. Lamb meat quality progress report number 1 . Preliminary results of an evaluation of effects of breed of sire on carcass composition and sensory traits of lamb. http://www.ars.usda.gov/sp2UserFiles/Place/54380530/ Publications/LambMeatQualityReportNumber1.pdf.

SIAP, 2017. Población de ovinos (cabezas). https://www.gob.mx/cms/uploads/attachment/file/412568/ Ovino 2017.pdf.

Snowder, G.D., Glimp, H.A. \& Field, R.A. 1994. Carcass characteristics and optimal slaughter weights in four breeds of sheep. J. Anim. Sci. 72(4): 932-937. doi: 10.2527/1994.724932x.

Swatland, H.J. 1991. Estructura y desarrollo de los animales de abasto: Estructura y propiedades de la carne. Acribia, Zaragoza, España. 458p. 